Artículo de Investigación

Apuntes del CENES

ISSN 0120-3053

Volumen $34-\mathrm{N}^{\circ} .60$

julio - diciembre 2015

Págs. 131-152

\title{
La inclusión social de la fuerza laboral en Colombia. ¿En contravía de lo sostenible?*
}

\section{Is social inclusion of workforce in Colombia in counter of what sustainability means?}

\section{A inclusão social da força de trabalho na Colômbia. É isto contrario do sustentável?}

Orlando Enrique Contreras** Aura Cecilia Pedraza Avella***

Carolina Herrera Gómez ****

Fecha de recepción: 3 de septiembre de 2014 Concepto de evaluación: 20 de enerode 2015 Fecha de aprobación: 25 de junio de 2015

\footnotetext{
Los autores son integrantes del Grupo de Investigación Finance \& Management adscrito a la Escuela de Estudios Industriales y Empresariales de la Universidad Industrial de Santander, Bucaramanga - Colombia.

** Ingeniero industrial y master of Business Administration. Profesor asistente de la Escuela de Estudios Industriales y Empresariales de la Universidad Industrial de Santander, Bucaramanga - Colombia. Coreo electrónico: ocontrer@uis.edu.co.

*** Ingeniera industrial y doctora en Ciencias Económicas. Profesora asociada de la Escuela de Estudios Industriales y Empresariales de la Universidad Industrial de Santander, Bucaramanga - Colombia. Correo electrónico: acecipe@uis.edu.co.

**** Ingeniera industrial de la Universidad Industrial de Santander, Bucaramanga - Colombia Correo electrónico: c.herreragomez15@gmail.com.
} 
La inclusión social de la fuerza laboral en Colombia. ¿En contravía de lo sostenible?

Orlando E. Contreras - Aura C. Pedraza Avella - Carolina Herrera Gómez

\section{Resumen}

Teniendo en cuenta la calidad del empleo como parte del concepto integral de sostenibilidad empresarial, el presente artículo demuestra a través del análisis de datos oficiales, que en Colombia se presentan diferencias considerables en cuanto a la inclusión social de los empleados de su sistema productivo. Las conclusiones obtenidas evidencian que los más excluidos poseen empleos de inferior calidad, como muestra de la precariedad laboral presente en el país. Esta situación indica que la revisión sistemática de sus políticas de sostenibilidad constituye una necesidad para el empresariado colombiano, particularmente en lo relacionado con el tipo de empleo que está brindando, considerando que el trabajo es un aspecto altamente influyente en la vida de los individuos.

Palabras clave: sostenibilidad, responsabilidad social empresarial, empleo, exclusión social, inclusión social, precariedad laboral.

Clasificación JEL: I3, J0, L2, M1.

\section{Abstract}

This article demonstrates through analysis of official data that in Colombia there are considerable differences regarding to social inclusion of its productive system employees, based on a framework that considers employment quality as an axe of corporate sustainability and object of social responsibility policies. Conclusions provide evidence to affirm that people with employments of inferior quality are the most excluded due to the consequences of a persistent labor scarcity in the country. The situation suggests that the Colombian business community needs a systematic review of sustainability policies, particularly in relation to the type of employment it is providing, given that work is a highly influential aspect of individuals life.

Keywords: sustainability, corporate social responsibility, employment, social exclusion, social inclusion, job instability. 


\section{Resumo}

Levando em conta a qualidade do emprego como parte do conceito integral da sustentabilidade empresarial, o presente artigo comprova através de uma análise de dados oficiais, que na Colômbia apresentam diferenças importantes sobre a inclusão social dos empregados do seu sistema produtivo. As conclusões obtidas mostram que os mais excluídos têm empregos de qualidade inferior como sinal da precariedade laboral presente no país. Este tipo de situação permite perceber que a revisão sistemática das suas políticas de sustentabilidade constitui uma necessidade para o empreendedorismo colombiano, especificamente com relação ao tipo de emprego que está sendo fornecido, levando em conta que o trabalho é um aspecto muito influente na vida das pessoas.

Palavras-chave: sustentabilidade, responsabilidade social empresarial, emprego, exclusão social, inclusão social, precariedade laboral. 
La inclusión social de la fuerza laboral en Colombia. ¿En contravía de lo sostenible?

Orlando E. Contreras - Aura C. Pedraza Avella - Carolina Herrera Gómez

\section{INTRODUCCIÓN}

El sistema productivo de un país, representado por sus empresas, es considerado como el eje fundamental del crecimiento económico; sin embargo, es claro que su accionar no siempre ha traído consecuencias positivas a la sociedad. Efectos perjudiciales en lo ambiental, social e incluso en lo económico, han ocasionado que hoy en día haya una demanda de la sociedad hacia un mayor compromiso de responsabilidad en las acciones empresariales. En este contexto surge el concepto de responsabilidad social corporativa o empresarial (RSE) que, según el informe de la reunión número 295 del Consejo de Administración de la Organización Mundial del Trabajo OIT (2006), «es el reflejo de la manera en que las empresas toman en consideración las repercusiones que tienen sus actividades sobre la sociedad». En este sentido, como expresan Lafuente, Viñuales, Pueyo y Llaría (2003, p. 7),

se dice que las organizaciones ejercen su responsabilidad social cuando prestan atención a las expectativas que sobre su comportamiento tienen los diferentes grupos de interés (tales como empleados, socios, clientes, comunidades locales, medioambiente, accionistas y proveedores), con el propósito último de contribuir a un desarrollo social y ambientalmente sostenible y económicamente viables.

Aproximaciones a la teoría y la práctica del concepto de la responsabilidad social empresarial se han esquematizado desde la contextualización de las funciones ejecutivas por parte de Barnard (1938), pasando por el pragmatismo de Levitt (1958), el utilitarismo de Friedman 
(1970), el desarrollo de la útil teoría del stakeholder por parte de Freeman (1990) y, por supuesto, aterrizando sobre lo que podría ser la primera relación entre la capacidad de competir de las organizaciones con la necesidad de «rendir cuentas» a su entorno, a través del marco de referencia denominado perspectiva, basada en los recursos de la firma, que fue planteado originalmente por Wernerfelt (1984) y más adelante retomado por Hart (2005) en un contexto práctico con un solo enfoque hacia la afectación medio-ambiental del entorno, que fue llamado más adelante responsabilidad social ambiental.

Sin embargo, posteriormente en la literatura se percibe la tendencia a consolidar conceptualmente los tópicos concernientes a bienestar social, ambiental y financiero con respecto a la demanda de la sociedad de que haya mayor responsabilidad en el actuar empresarial, y es allí en donde se contextualiza la sostenibilidad1. A manera de referencia, vemos como, por ejemplo, McWilliamS, Siegel y Wright (2006) hacen una alineación de conceptos entre responsabilidad social corporativa y estrategia, que a la postre fue retomada por Porter y Kramer (2006 y 2011) en donde, entre otras cosas, ponen de relieve la necesidad de profundizar en el tema y la inquietud despertada por miembros de la alta gerencia de grandes organizaciones sobre esta fuerte idea. Ya no era un problema de retribuirle de alguna forma a la sociedad o al medio ambiente por la huella que se había dejado por el accionar desmesurado de las empresas, era un problema de supervivencia que en el futuro iba a ser coherente con las acciones que en todo nivel se desarrollan en el ámbito organizacional. La sostenibilidad había hecho su arribo oficial como concepto y como uno de los paradigmas dominantes en el ámbito de la formulación e implementación de una estrategia orientada a garantizar la supervivencia de la sociedad y, por definición, de los negocios.

A partir de allí, multiples contribuciones hicieron evidente dicha tendencia tanto en términos académicos como prácticos, hasta el punto que, de manera inevitable, algunos autores como Pinillos y Fernández (2011) llegaron a sostener que mas allá de los conceptos de responsabilidad social y filantropía empresarial, el marco de referencia actual debe tener una orientación efectiva hacia la sostenibilidad en las empresas (sostenibilidad empresarial), la cual está asociada directamente a la creación de valor integral a largo plazo; es decir, que este debería ser el referente conceptual de ahora en adelante. De esta forma se concluye que la tendencia de emprender y administrar negocios que impacten positivamente el medio ambiente o la sociedad es totalmente compatible y coherente (de hecho casi sinérgico) con los legítimos intereses de creación de

Viene del inglés Sustainability. En español también referenciado como sustentabilidad o sustentable, o ambos. 
La inclusión social de la fuerza laboral en Colombia. ¿En contravía de lo sostenible? Orlando E. Contreras - Aura C. Pedraza Avella - Carolina Herrera Gómez

riqueza financiera de los empresarios, $\mathrm{y}$ es por ello que nos referiremos a la sostenibilidad empresarial como concepto integrador.

Ahora, teniendo en cuenta que los empleados hacen parte de esa sociedad que las empresas deben impactar positivamente, aparece el concepto de sostenibilidad en el ámbito interno de las organizaciones. «Las prácticas responsables en lo social que inciden en el ámbito interno de la organización afectan, en primer término, a los trabajadores y se refieren a cuestiones relativas a la gestión e inversión en recursos humanos, a la salud y seguridad en el trabajo y a la gestión del cambio y mantener un clima laboral sano» (European Council, 2002, p. 8). Asimismo la norma de Sostenibilidad ISO 26000 (2010) afirma que es en la creación de puestos de trabajo, así como en los salarios y las compensaciones, que se encuentra una de las principales formas en que las empresas pueden contribuir a la sociedad.

Es así como de manera integral, la idea de lo sostenible ha venido envolviendo prácticamente todos los ámbitos de una organización, incluyendo también a sus empleados a través de las acciones y decisiones que la compañía ejecuta día a día y que afecta o son afectadas por este importante stakeholder. El entendimiento del impacto (positivo o negativo) que genera en el performance organizativo el estado integral de su recurso humano es un hecho que se ha relacionado ampliamente en la literatura y que llega a su máximo apogeo con las conclusiones que Spreitzer y Porat (2012) esquematizaron a la luz de lo que ellos llamaron prosperidad empresarial en relación directa de causalidad frente a la misma prosperidad de sus propios empleados.

Y es precisamente en este punto en donde se encuentra claramente la conexión (o mejor, desconexión) dialéctica y conceptual que hace evidente el hecho de que la exclusión social genera un efecto contrario al desarrollo sostenible, toda vez que este último es definido universalmente como «aquel que satisface las necesidades del presente sin comprometer la capacidad de las generaciones futuras de satisfacer sus propias necesidades» (Informe Brundtland ONU, 1987). Asi las cosas, y a manera de ejemplo, en la medida en que las personas posean empleos de baja calidad, es claro que con la incompleta satifacción de sus necesidades, no solo se está atentando sobre su desarrollo presente, sino que se descuida el desarrollo de sus familias y por definición, la capacidad de satisfacer las necesidades de sus futuras generaciones. En este sentido, autores como Earnes y Adebowale (2012), Dempsey, Bramley, Power y Brown (2012), y Fritz y Koach (2014), así como sendos estudios de la International Labour Organization (Poschen, 2012) y la United Nations Research Institute for Social Development (Dugarova \& Lavers, 2014) lo han puesto de manifiesto y en muchos casos han demostrado la existencia de dicha corre- 
lación que, de la misma manera, representa el centro de nuestra discusión.

Es de esperarse entonces que empresas con buenas prácticas en la vía de lo sostenible en su ámbito interno, contribuyan a la sociedad con empleados no excluidos socialmente, y que aquellas que no están comprometidas con mantener buenas prácticas internas sostenibles, generen resultados contrarios. Por tanto, se esperaría que así como lo afirma el European Council (2002), el empleo fuera una salvaguardia contra la exclusión social.

Dado lo anterior, se puede afirmar que una de las consecuencias de la sostenibilidad empresarial hace alusión a una mejor calidad de vida de los trabajadores, entendiendo esta como

la percepción que un individuo tiene de su lugar en la existencia, en el contexto de la cultura y del sistema de valores en los que vive y en relación con sus objetivos, sus expectativas, sus normas, sus inquietudes. Se trata de un concepto muy amplio que está influido de modo complejo por la salud física del sujeto, su estado psicológico, su nivel de independencia, sus relaciones sociales, así como su relación con los elementos esenciales de su entorno (WHO, 1995, p. 41).

En relación con este concepto, como define Pedraza (2012) basado en Amartya Sen (1985, 1993 y 2000), el estado de exclusión social en un individuo puede definirse como el entrelazamiento de privaciones relevantes, relativas a la sociedad donde se vive ${ }^{2}$ y se relacionará directamente con la naturaleza de la vida que este lleva en comparación con el resto de individuos de su sociedad, yendo más allá del ingreso o las posesiones con las que cuenta y considerando su capital humano, capital social e integración política, espacial y laboral ${ }^{3}$. Según Sen (2000), el bienestar

2 Como noción analítica, el término exclusión social parece haberse acuñado originalmente en Francia por Rene Lenoir en su libro de 1974, Los excluidos: un francés de diez (Les Exclus: Un français sur dix), para referirse a varias categorías de personas catalogadas como problemas sociales y quienes no gozaban de la protección de la seguridad social. Hoy en día existe un sinnúmero de definiciones de la exclusión social pero, en general, siempre se ve como un estado de malestar y discapacidad. Para Jordan (1996), por ejemplo, este concepto se asocia a la falta de poder individual de algunas personas para acceder a recursos comunes a toda la ciudadanía, que se convierte en un obstáculo estructural en el nivel social. Bhalla y Lapeyre (1999) definen exclusión social como un proceso que hace que individuos o grupos, quienes residen geográficamente en una sociedad, no participen en las actividades normales de los ciudadanos de esa sociedad. Siguiendo a Castel (1997), "serían excluidos aquellos que no participan de los intercambios regulados y que por tal razón la sociedad no reconoce en ellos una contribución significativa al ordenamiento social, así fuese en el marco de unos intereses contrapuestos o disímiles". En últimas, como dice Sen (2000, p.9) "El lenguaje de exclusión es tan versátil y adaptable que hay mucha tentación a disfrazar cualquier privación como un caso de exclusión social. Hay, me temo, alguna evidencia en la amplia -y rápidamente crecienteliteratura sobre exclusión social de que el lenguaje ha sobrepasado las ideas creativas implicadas".

3 Esto corresponde a una definición multidimensional de la exclusión social que es la más aceptada en los últimos tiempos. Esta tendencia es impulsada principalmente por organismos enfocados en la formulación de políticas sociales, tales como la Comisión Europea (2001) y el Instituto de Estudios Laborales de la Organización Internacional del Trabajo, y su principal defensor es el premio Nobel Amartya Sen. Las otras dos perspectivas de la exclusión social que han sido relevantes en el estudio del fenómeno son la anglosajona y la francesa republicana, que tienen explicaciones monocausales de la exclusión social, la primera centrada en la pobreza y la segunda en el rompimiento de los lazos sociales. 
de un individuo no estará determinado por los bienes que posee sino por sus funcionamientos, es decir, por las cosas que una persona ha logrado ser y hacer en su vida. Esta aproximación teórica de la exclusión social, dice Sen (2000), tiene sus raíces en el pensamiento aristotélico, consignado por ejemplo en Ética a Nicodemo donde se asevera que todo individuo vive en una «inescapable vida social».

Las personas pueden sufrir simultáneamente de privaciones en algunos o todos los funcionamientos de las dimensiones relevantes que son la física, de capital humano, económica, de capital social, política y labor ${ }^{4}$ en un punto en el tiempo. En una descripción elemental de los estándares de vida, la dimensión física incluirá funcionamientos de localización e infraestructura, la de capital humano aquellos de salud y educación, la dimensión económica reunirá información sobre ingresos y patrimonio, la de capital social conjugará funcionamientos de las relaciones interpersonales, la política dará cuenta del ejercicio de derechos $\mathrm{y}$ ciudadanía $\mathrm{y}$, finalmente, la dimensión laboral señalará la participación del individuo en el mercado de trabajo. La independencia de esta última categoría, que algunos autores como De Haan (1999) incluyen en la dimensión económica, tiene su validez en el hecho que las sociedades capitalistas se han fundamentado en torno al trabajo y los individuos permanecen gran parte de su vida en ambientes laborales; emplearse no es solo garantía de un nivel de ingresos sino que transforma la ubicación física del individuo, sus posibilidades de adquirir capital humano y su cohesión social y política.

Dado su carácter multidimensional, el estado de exclusión social puede ser causado por cualquiera de las privaciones relevantes, ya que, como sustentan Rubio y Monteros (2002), cada una de estas situaciones de desventaja se retroalimenta con las otras. Sin embargo, la dimensión laboral podría tener mayor probabilidad de dar inicio a la acumulación de desventajas (Pedraza, 2010, p.19). Esto teniendo en cuenta que la participación en el mundo laboral proporciona, además de la seguridad del ingreso, un espacio de desarrollo, un estatus y una identidad $^{5}$. El trabajo supone una oportunidad para la autorrealización y es un instrumento de socialización (Pedraza, 2011). Como señaló Alfred Marshall (1938, p. 566): «Nada le importa al vendedor de ladrillos que éstos vayan a emplearse para construir un palacio o una alcantarilla, pero sí le importa mucho al vendedor de trabajo, que se dispone a realizar una tarea de una determinada

\footnotetext{
$4 \quad$ Tal como lo comenta Silver (1995), en la literatura se habla de individuos excluidos de espacios tan disímiles como: "un sustento; empleo permanente o seguro; ganancias; propiedad, crédito o tierra; vivienda; niveles de consumo mínimos o que prevalecen; educación, habilidades y capital cultural; el Estado de Bienestar; ciudadanía e igualdad legal; participación democrática; bienes públicos; la nación o raza dominante; familia y sociabilidad; humanidad, respeto cumplimiento y entendimiento". Por tal motivo, es útil establecer cuáles son las dimensiones sociales elementales.

5 En palabras del grupo de investigación Milenium 3, en un trabajo para CAI e INAEM (2007, p. 113), "el trabajo es la principal vía de integración social y personal”.
} 
dificultad, que el lugar en el que ha de realizarla sea saludable y agradable o que sus compañeros sean los que a él le gustaría tener».

Sin embargo, mucho se ha hablado en los últimos años acerca de la precariedad laboral, dando lugar al concepto de trabajadores pobres (o working poors, por su nombre más común en inglés). Como reporta Gundogan, Bicerli y Aydin (2005, p.7),

aproximadamente un $49.7 \%$ de los trabajadores del mundo (y un poco más del $58.7 \%$ de los trabajadores de los países en desarrollo) no están ganando suficiente para que ellos y sus familias superen la línea de la pobreza de los dos dólares diarios, y un $19.7 \%$ de las personas empleadas a nivel mundial (y aproximadamente un $23.3 \%$ de aquellos de los países en desarrollo) están viviendo con menos de un dólar diario.

En este contexto cobra especial atención lo dicho por Atkinson (1998) acerca de que el empleo garantiza la inclusión social dependiendo de la calidad del empleo ofrecido.

La situación anterior indica que un buen número de empresas han descuidado las políticas sostenibles relacionadas con el bienestar de sus empleados y al brindar empleos de mala calidad están contribuyendo a su exclusión social. Es así como surge la relación entre la inclusión de los trabajadores a la sociedad y las prácticas de sostenibilidad empresarial. Como lo expresa la ISO 26000:33, «las prácticas laborales tienen un impacto importante en respeto del Estado de derecho y en el sentido actual de la justicia en la sociedad; las prácticas laborales socialmente responsables son esenciales para la justicia social, la paz y la estabilidad». Es por ello que la propuesta que representa un abordaje mas coherente con dicha problemática proviene del campo del management, pues cada día se va propiciando con más intensidad un énfasis hacia el concepto de organización responsable, en donde temas como la ética, los valores, la equidad y el respeto por las diferencias entre las personas deberían permear el sistema decisional de los empleadores a fin de propiciar entornos más tolerantes y, a la vez, más productivos. La lógica nos sugiere, por tanto, integrar los principios de la sostenibiidad con la gerencia de recursos humanos (Ehnert, Harry \& Zink, 2014).

\section{LA SOSTENIBILIDAD COMO DRIVER DE LA GESTIÓN DE RECURSOS HUMANOS}

Apelar a prácticas de gerenciamiento de recursos humanos que le apunten a la consolidación de organizaciones que tengan la mira en la aplicación legítima de políticas de responsabilidad social y sostenibilidad se ha convertido en un aspecto fundamental en la forma de hacer negocios hoy en día, y a la vez en un referente de la literatura gerencial de los últimos años (Bourdeau \& Ramstad, 
2005; Tarique \& Schuler, 2012; Lis, 2012). De hecho, las evidencias obtenidas a través de sendos estudios demuestran que dichas prácticas están alineadas con un mejor desempeño empresarial y representan desafíos que la gerencia deberá enfrentar en su futuro (Stone \& Deadrick, 2015) para lograr un entorno de prosperidad.

A manera de ejemplo, vemos el surgimiento del concepto rendimiento sostenible (sustainable performance), el cual consiste en la aplicación de cuatro «mecanismos» que permiten dicha prosperidad: (1) proveer empoderamiento para la toma de decisiones; (2) compartir información clave; (3) minimizar la «incivilidad» organizativa; $y$ (4) ofrecer feed-back sobre el desempeño. En un intento por dejar en claro que es a través de las personas que se obtienen resultados sobresalientes en el campo de la sustentabilidad (especialmente en lo que al impacto ambiental se refiere), es también de especial importancia el trabajo de recopilación de visiones y aportes que realizaron Renwick, Redman y Maguire (2013), que, a la postre es bautizado como la gerencia «verde» de los recursos humanos, haciendo alusión a las prácticas usuales en la dirección de las personas que deberían ser parte del día a día de gerentes y ejecutivos, en la búsqueda de lo que ellos llamaron una integración entre la gerencia de los recursos humanos con la gerencia ambiental.

Asimismo, a través de la investigación de Jones (2010) se puede encontrar un aporte enorme a la validación del fenómeno de los programas de intercambio social y voluntariado en las organizaciones orientados al servicio a la comunidad por parte de sus colaboradores. Adicional al completo recuento de los aportes que otros autores han realizado al respecto, se logra corroborar la efectividad de este tipo de prácticas en función de aspectos tales como la lealtad de los empleados hacia sus empleadores, debida en gran medida, al orgullo de pertenecer a estas organizaciones. También fueron objeto de este mismo estudio variables como la retención de personal, el clima laboral y la sensibilidad hacia su sentimiento con respecto a su compensación; todos ellos como respuesta positiva a las prácticas socialmente responsables de las organizaciones analizadas.

Por otra parte, a través de conclusiones más recientes de Jones, Willnes y Madey (2011) también se pudo encontrar una correlación directa entre los niveles de eficacia de las funciones inherentes a la incorporación de personas en las organizaciones y la percepción que el público tiene sobre ellas, en términos de lo que los autores definieron como el desempeño social responsable6; es decir, que se pudo comprobar una sensibilidad directa e importante entre los resultados de los procesos de reclutamiento de personal en este tipo de organizaciones como consecuencia de la positiva percepción de los candidatos hacia estas, en términos de sus prácticas de responsabilidad social y sostenibilidad. A la postre, influyendo en la motivación 
de los candidatos no solo a pertenecer a dichas organizaciones, sino a generar un compromiso real y a largo plazo con ellas a través de su trabajo.

Desde la perspectiva práctica, también se encuentra evidencia de algunas políticas, prácticas y decisiones que influyen positivamente en los resultados relativos al sistema gerencial de talento humano en organizaciones que actúan en el marco de lo sostenible. Sobre esto existen ejemplos de compañías, como Ben \& Jerry's como muestra de innovación social; Timberland LLC al utilizar su reputación como apoyo directo a su marca; Marks and Spencer con el gerenciamiento sostenible de su cadena de abastecimiento; y Florida Ice \& Farm, como ejemplo latinoaméricano a través del logro de su programa «agua neutral». Todos ellos, llevados a la literatura por Page y Katz (2012), Blaisdell (2012), Brokaw (2012) e Ickis, Prado y García-Rada (2012) respectivamente, sirven para ilustrar lo deseado. Todos con importantes elementos en común que validan los postulados básicos de la sostenibilidad: se refieren a empresas exitosas con altos niveles en su reputación como marcas y como empresas en sí mismas que mantienen un equilibrio con su sistema gerencial de recursos humanos.

Estos postulados constituyen, sin duda alguna, la evolución refinada de lo que alguna vez definiera Ulrich (1997) como la «clave del éxito» de los ejecutivos que dirigen personas para afrontar los retos de la competitividad que (en ese momento y hoy en día) se vislumbran en el horizonte. En otras palabras, y apelando a los estudios desarrollados por la Ohio State University en 1957 y recogidos por Fiedler y Chemers (1985), un líder (para nuestro caso, un gerente de recursos humanos) a través de su conducta es capaz de hacer que una empresa genere los resultados que sus empleados son capaces (competencia) $\mathrm{y}$ tienen deseo (motivación) de generar.

De esta forma y teniendo en cuenta los antecedentes expuestos, el presente estudio propone como objetivo analizar la relación existente entre las condiciones laborales de los ocupados en Colombia y su exclusión o inclusión social, como parte de las políticas de sostenibilidad interna de los empleadores.

\section{METODOLOGÍA}

Se aplicó un análisis econométrico a la información obtenida a través de la Encuesta de Calidad de Vida 2003 y 2008, realizada por el Departamento Administrativo Nacional de Estadística (DANE), mediante el software Stata 11.1. Se toma únicamente la población urbana, teniendo en cuenta la gran diferenciación que existe entre las ocupaciones que allí se crean frente a aquellas de la zona rural.

La Encuesta de Calidad de Vida del DANE cuantifica y caracteriza las condiciones de vida de la población colombiana, analiza 
variables relacionadas con condiciones de vivienda, (material de paredes, pisos y acceso a servicios públicos) y tenencia de esta. Adicionalmente, indaga por características específicas de los miembros del hogar, como educación, salud, cuidado de los niños, fuerza de trabajo, gastos e ingresos, entre otros. En el caso específico de la versión del año 2008, se incluye información adicional sobre seguridad alimentaria y nutricional, así como sobre la tenencia y el uso de tierras en el área rural.

Hasta 2008 se habían realizado 3 encuestas, es decir, una cada 5 años, para los periodos 1997, 2003 y 2008. $\mathrm{Su}$ aplicación es fruto de un trabajo de campo realizado en 9 regiones del país, y gracias a la riqueza de información obtenida, con base en ella es posible calcular indicadores para factores tales como vivienda, salud, educación, recreación y cultura, productividad $\mathrm{y}$ empleo, calidad de vida, y riesgo.

Para efectos del presente estudio, en primer lugar se caracterizó la población según su actividad económica, escogiendo a quienes tenían una ocupación en el momento de la medición, según la definición de desempleo contextualizada por el DANE.
Posteriormente, los trabajadores fueron clasificados según la calidad de su empleo. Con el fin de evaluar esta característica de las ocupaciones, a partir de diversos factores que podrían generar ventajas o desventajas para los empleados, se construyó un índice con base en la guía dada por Farné (2003). Este autor calcula el indicador de calidad del empleo a partir de los siguientes criterios: i. Los ingresos laborales sumando los salarios y los pagos en especie (100 puntos para ingresos superiores o iguales al salario mínimo vigente y 0 puntos para ingresos inferiores a dicha cantidad142). ii. La afiliación a seguridad social (100 puntos si está afiliado a pensión y salud, 50 si está afiliado sólo a alguno de los dos y 0 si no está afiliado a ninguno de los dos sistemas), y iii. La jornada laboral (100 puntos si trabaja hasta 48 horas semanales y 0 puntos si trabaja más). Sin embargo, a diferencia de Farné (2003), en este estudio para construir el índice de calidad del empleo no se asignaron pesos a los tres factores analizados a criterio del investigador, sino que para esta tarea se utilizó la técnica estadística de análisis de correspondencias múltiples ACM, que es un análisis de componentes principales ACP pero diseñado para variables binarias ${ }^{7}$. Esta decisión se toma dada la utilidad del ACM para

\footnotetext{
Como mencionan Asselinn y Anh (2008, p. 2), el ACP "esencialmente consiste en construir una secuencia de combinaciones lineales no correlacionadas (ortogonales) y normalizadas de las $\mathrm{k}$ variables de entrada, agotando la variabilidad completa del conjunto de variables de entrada, llamado la "varianza total" y definida como la traza de la matriz de covarianza, es decir la suma de las k varianzas. El proceso de reducción descrito, equivalente a la identificación de los valores y vectores propios, corresponde geométricamente a un cambio en el sistema de ejes cartesianos (translación y rotación) del espacio euclidiano de dimensión k. Básicamente el ACM es un proceso de ACP que usa la métrica chi cuadrado $X^{\wedge} 2$ en lugar de la Euclidiana". (Asselin \& Anh, 2008, p. 4).
} 
hacer escalamientos óptimos cuando el objetivo es captar la máxima correlación entre variables. Posteriormente, se obtuvo un indicador binario de calidad del empleo llamando 1 a aquellos con empleos de peores condiciones, tomando como punto de corte la media del indicador, aprovechando que el ACM entrega valores normalizados.

En tercer lugar se calculó el grado de exclusión social de los ocupados, mediante un índice multidimensional, a partir de la siguiente ecuación:

$$
e s_{i}=\sum_{x=1}^{c}\left[w_{x} * d\left(f_{i x}\right)\right]
$$

Siendo esta una combinación lineal de las privaciones relevantes acumuladas por $\mathrm{i}$, que en total son un número $\mathrm{c}, \mathrm{d}(\mathrm{fix})$ es la privación de la persona $i$, dado su nivel del funcionamiento $\mathrm{fx}, \mathrm{y}$ wx el aporte a la exclusión social de la respectiva privación que, en este caso, se calcularon nuevamente a través de un ACM. En este caso, la ventaja de usar estos pesos factoriales es que se da mayor relevancia a las privaciones sufridas por la gran mayoría de los individuos de la sociedad.

Finalmente se validaron estadísticamente las diferencias entre la exclusión social de los trabajadores con empleos de buena y mala calidad.

\section{RESULTADOS}

En la Tabla 1 se muestran los resultados de calidad del empleo urbano para los años 2003 y 2008 en Colombia, encontrándose que cerca del $65 \%$ de las ocupaciones son de mala calidad.

Los resultados muestran diferencias en la calidad de empleo ofrecido, donde más de la mitad de las ocupaciones brindadas son de mala calidad. Esto evidencia que las políticas de responsabilidad social empresarial interna están siendo ignoradas por una buena proporción de las empresas en Colombia o, si las tienen, que no están siendo muy efectivas.

Ahora bien, se quiso estudiar la exclusión social de los trabajadores urbanos en Colombia, y para medirla se construyó un indicador multidimensional que, siguiendo a De Haan (1999) pero limitados por la información de las encuestas, tuvo en cuenta las desventajas por las privaciones que se muestran en la Tabla $2^{8}$. Aunque se quiso incluir alguna privación relacionada con la vida política de los individuos, esto no fue posible por la ausencia de información disponible, sobre todo para el año 2008.

$8 \quad$ La medición de la exclusión social para la población en general contempla también la dimensión laboral. Sin embargo, como en este caso solo se está analizando la población ocupada, no se tiene en cuenta el indicador de desempleo. La precariedad laboral tampoco se incluye en el cálculo del indicador multidimensional, porque es precisamente la diferencia entre la población por esta característica la que se pretende estudiar a continuación con mayor detalle. 
La inclusión social de la fuerza laboral en Colombia. ¿En contravía de lo sostenible?

Orlando E. Contreras - Aura C. Pedraza Avella - Carolina Herrera Gómez

Tabla 1. Empleo de baja calidad en Colombia

\begin{tabular}{|c|c|c|}
\hline & $\mathbf{2 0 0 3}$ & $\mathbf{2 0 0 8}$ \\
\hline Total & $64.16 \%$ & $63.64 \%$ \\
\hline
\end{tabular}

Fuente: elaboración propia con base en ECV (2003) y ECV (2008).

Tabla 2. Privaciones relevantes en Colombia

\begin{tabular}{|c|c|c|}
\hline DIMENSIÓN & CATEGORÍAS & INDICADORES DE PRIVACIÓN \\
\hline \multirow{3}{*}{ Física } & $\begin{array}{l}\text { Infraestructura } \\
\text { de la vivienda }\end{array}$ & $\begin{array}{l}\text { - Tipo de vivienda } \\
\text { - Material de las paredes } \\
\text { - Material de los pisos } \\
\text { - Hacinamiento }\end{array}$ \\
\hline & $\begin{array}{l}\text { Localización } \\
\text { de la vivienda }\end{array}$ & $\begin{array}{l}\text { - Riesgo inminente } \\
\text { - Sitio peligroso en la vecindad } \\
\text { - Seguridad del barrio }\end{array}$ \\
\hline & $\begin{array}{l}\text { Servicios } \\
\text { domiciliarios }\end{array}$ & $\begin{array}{l}\text { - Disponibilidad de servicios domiciliarios } \\
\text { - Tipo de sanitario } \\
\text { - Abastecimiento de agua } \\
\text { - Combustible utilizado }\end{array}$ \\
\hline \multirow[t]{2}{*}{ Capital humano } & Salud & $\begin{array}{l}\text { - Estado general de salud } \\
\text { - Enfermedad crónica }\end{array}$ \\
\hline & Educación & $\begin{array}{l}\text { - Analfabetismo } \\
\text { - Máximo nivel educativo alcanzado }\end{array}$ \\
\hline \multirow[t]{2}{*}{ Economía } & $\begin{array}{l}\text { Dependencia } \\
\text { económica }\end{array}$ & $\begin{array}{l}\text { - Número de personas por ocupado en el } \\
\text { hogar }\end{array}$ \\
\hline & Riqueza & $\begin{array}{l}\text { - Bienes acumulados en el hogar } \\
\text { - Consumo de tres comidas diarias }\end{array}$ \\
\hline \multirow[t]{2}{*}{ Capital social } & Contactos sociales & $\begin{array}{l}\text { - Sin lazos familiares primarios en el hogar } \\
\text { - Nuevo en el municipio donde habita }\end{array}$ \\
\hline & Bienestar del hogar & $\begin{array}{l}\text { - Discapacitados en el hogar } \\
\text { - Año difícil para el hogar }\end{array}$ \\
\hline
\end{tabular}

Fuente: elaboración propia

Una vez fueron establecidos indicadores binarios para todas las privaciones señaladas, estos se integraron en un índice multidimensional construido a partir de un análisis de correspondencias múltiples, además se construye una variable binaria de exclusión social de acuerdo con el punto medio del intervalo en que está agrupado dicho indicador. La Tabla 3 muestra los resultados obtenidos con la información de los años 2003 y 2008 acerca de la exclusión social de los ocupados urbanos según la calidad del empleo con el que contaban, evidenciando que la precariedad laboral está relacionada con la exclusión social. 
Tabla 3. Exclusión social en Colombia según la calidad del empleo

\begin{tabular}{|c|c|c|c|c|}
\hline & \multicolumn{2}{|c|}{ PORCENTAJE DE EXCLUIDOS SOCIALMENTE } \\
\hline & \multicolumn{2}{|c|}{$\mathbf{2 0 0 3}$} & \multicolumn{2}{c|}{$\mathbf{2 0 0 8}$} \\
\hline & $\begin{array}{c}\text { Empleo } \\
\text { baja } \\
\text { calidad }\end{array}$ & $\begin{array}{c}\text { Empleo } \\
\text { alta } \\
\text { calidad }\end{array}$ & $\begin{array}{c}\text { Empleo } \\
\text { baja } \\
\text { calidad }\end{array}$ & $\begin{array}{c}\text { Empleo } \\
\text { alta } \\
\text { calidad }\end{array}$ \\
\hline Total & $57.39 \%$ & $30.67 \%$ & $56.36 \%$ & $29.82 \%$ \\
\hline
\end{tabular}

Fuente: elaboración propia con base en ECV (2003) y ECV (2008).

\section{DISCUSIÓN DE LOS RESULTADOS}

Los resultados obtenidos muestran que tanto para el año 2003 como para el 2008, los individuos que trabajan en los empleos categorizados como de baja calidad tienen mayor exclusión social que aquellos que ocupan los de mejor calidad (ver Tabla 3).

La significancia estadística de esta diferencia fue corroborada mediante un pareo por máxima verosimilitud (ver Tabla 4). Esta técnica permite verificar la exclusión social para individuos con las mismas características ubicados en empleos de diferente calidad. Una vez más, se encuentran diferencias entre los porcentajes de exclusión social según las características de los individuos que prevalecen sobre las diferencias por calidad del empleo, y son persistentes del año 2003 al 2008.

Tabla 4. Pareos por máxima verosimilitud de las diferencias de exclusión entre ocupados.

\begin{tabular}{|c|c|c|}
\hline \multicolumn{3}{|c|}{ Grupo de control: empleos de mejor calidad } \\
\hline MUESTRA & $\mathbf{2 0 0 3}$ & $\mathbf{2 0 0 8}$ \\
\hline Efecto Promedio (ATT) & 0.075265475 & 0.375091654 \\
\hline $\begin{array}{c}\text { Intervalo de confianza }- \\
\text { muestreo repetido } \\
\text { (bootstrapping) }\end{array}$ & {$[0.05651950 .094011]$} & {$[0.42314480 .3270385]$} \\
\hline
\end{tabular}

Fuente: elaboración propia con base en ECV (2003) y ECV (2008).

Así las cosas, la calidad de los trabajos ofertados en Colombia no parece contribuir a garantizar mejores condiciones a sus colaboradores, dándole la razón a Atkinson (1998, p. v) al afirmar que el empleo solo garantiza la inclusión social cuando es de buena calidad.
Estos resultados darían evidencia de que en Colombia las políticas de sostenibilidad empresarial interna son inexistentes o no están siendo totalmente efectivas, puesto que se promueve la precariedad laboral con consecuencias negativas en la inclusión social de los trabajadores. 
La inclusión social de la fuerza laboral en Colombia. ¿En contravía de lo sostenible?

Orlando E. Contreras - Aura C. Pedraza Avella - Carolina Herrera Gómez

\section{EL TELETRABAJO, UN EJEMPLO DE PRÁCTICA LABORAL «INCLUSIVA»}

Muy a pesar del debate existente entre la adopción de tecnologías y la existencia de politicas de inequidad en el desarrollo de las regiones y países, abordado críticamente por Bogliacino (2014), una alternativa se destaca entre muchas para demostrar que bien concebido, este esquema podría representar una muestra clara de práctica sostenible de recursos humanos y un promotor de políticas de inclusión social en la medida en que las condiciones laborales mejoren para los individuos insertos en el mercado laboral colombiano. Nos referimos al teletrabajo, tal cual como lo aseguran Contreras y Rozo (2015) en su trabajo reflexivo.

Partimos de la base de que en los últimos años es indiscutible la labor que el Estado y el empresariado colombiano han estado desarrollando en la búsqueda de incorporar una filosofía de trabajo fuertemente vinculada al uso de las TIC, a fin de apuntar a una mejora paulatina del indicador de la productividad, del desempleo y, por ende, de la inclusión laboral en el país.

De hecho, se evidencia el vínculo con el presente trabajo, gracias a conclusiones previas sobre la relación directa entre el uso de las TIC y el desempeño de las empresas en Colombia; en donde, a manera de ejemplo, algunos estudios como el de Botello, Pedraza y Contreras (2014), luego de obtener resultados de correlación directa entre productividad y adopción de TIC en las organizaciones, también recomiendan la promoción de políticas públicas y empresariales orientadas a ampliar la cobertura de las diferentes tecnologías de la información y comunicación en el sector servicios de Colombia.

Así las cosas, esta temática se aborda a partir de un enfoque a lo formalmente establecido desde la perspectiva oficial, en donde lo más tangible es definitivamente el énfasis puesto sobre la adopción de la figura del teletrabajo como opción laboral formal, lo cual ha sido materializado por el Gobierno nacional a través de mecanismos que formalizan y estimulan la práctica en mención: la Ley ${ }^{9}$ del Teletrabajo y su posterior decreto reglamentario ${ }^{10}$, la creación del «Pacto por el teletrabajo», la vinculación de diferentes entes territoriales, empresas, universidades y personas, a las iniciativas impulsadas por los ministerios ${ }^{11}$, para crear una masa crítica decente que reciba correctamente el mensaje y promueva su implementación. Estas son interesantes

\footnotetext{
Ley 1221 de 2008.

10 Decreto 884 de 2012.

11 Tales como la Comisión Asesora de Teletrabajo, La Red Nacional de Fomento al Teletrabajo, la firma de convenios internacionales para lograr aprendizaje cruzado en torno al fenómeno y la edición del Libro blanco abc del teletrabajo, que viene siendo el cuerpo del conocimiento útil para "facilitar a las empresas la implementación de proyectos piloto de teletrabajo a través de una metodología ajustada al contexto colombiano".
} 
iniciativas que han resultado en una normativa interesante pero sin un sentido claro de su alcance o sus implicaciones, las cuales despiertan necesariamente algunas inquietudes relacionadas con su medición, monitoreo y control.

De cualquier forma, y muy a pesar de que la medición del impacto positivo o negativo de esta practica laboral es incipiente y difícil; se puede afirmar sin temor a la equivocación, que la intención es loable y que con un enfoque adecuado, se puede llegar efectivemente a un aporte directo al desarrollo de Colombia en términos de la percepción de inclusión social de los trabajadores. Es decir, que se podría estar trabajando en la vía de lo ambiental, social y económico-productivo (sostenibilidad).

\section{CONCLUSIONES}

La sostenibilidad empresarial, vista como el conjunto de acciones que buscan repercusiones positivas sobre la sociedad, incluye el cuidado que deben tener las organizaciones de brindar empleos de buena calidad. Esta sostenibilidad empresarial interna incluirá entonces políticas relacionadas con estructuras salariales equitativas, pero también aquellas relacionadas con condiciones no monetarias de las ocupaciones. Teniendo en cuenta que el medio laboral es parte fundamental de los espacios de inclusión de los individuos a la sociedad, las consecuencias de la sostenibilidad interna se reflejarán en el bienestar de la población.

Los resultados para Colombia muestran que las políticas de sostenibilidad interna en las organizaciones son inexistentes o no están siendo totalmente efectivas, puesto que para los años 2003 y 2008 se puede encontrar cerca de un 30 $\%$ de personas con empleos de mala calidad. La mayor exclusión social de los trabajadores en ocupaciones de baja calidad frente a aquellos que se desempeñan en las mejores ocupaciones, genera una mayor relevancia de la revisión de estas políticas. Las empresas deben ser conscientes de que al ignorar la responsabilidad que ellas tienen con sus empleados, están condenando a buena parte de ellos a ser excluidos de la sociedad.

La situación planteada permite conjeturar que la revisión sistemática de las políticas de sostenibilidad de las organizaciones de países en vías de desarrollo como Colombia constituye una necesidad imperante en la agenda de desarrollo empresarial y competitivo, particularmente en lo relacionado con el tipo de empleo que se está brindando en su mercado laboral. Esto se da teniendo en cuenta que el trabajo es indudablemente un aspecto altamente influyente en la vida de los individuos, pues dedican a él gran parte de sus vidas y muchas veces constituye su única fuente de ingresos y de integración social. 
La inclusión social de la fuerza laboral en Colombia. ¿En contravía de lo sostenible?

Orlando E. Contreras - Aura C. Pedraza Avella - Carolina Herrera Gómez

Parte de la discusión que debe darse en escenarios posteriores y que puede derivarse del presente análisis, es la afectación que en la vía del negocio (es decir, sobre sus resultados financieros), las organizaciones están obteniendo a partir del descuido de prácticas de sostenibilidad interna. Prematuramente se piensa que la relación entre estas dos variables debería ser positiva y directa, es decir, que a medida que una empresa fomente la aplicación de prácticas de sostenibilidad interna en su accionar, se verá un impacto positivo generado sobre los resutados de su negocio, y viceversa. Sin embargo es necesario validarlo con un mayor nivel de detalle, lo cual representa interesantes oportunidades de investigación futura en el ámbito de los estudios exploratorios, análisis de impacto y estudios de caso de tipo pedagógico e investigativo que abran la puerta del análisis y del aporte a la praxis administrativa.
Una aproximación válida a este último punto, podría venir desde el campo de la gerencia, en donde, tal y como lo plantearon Contreras, Vecino y Pedraza (2013), la toma de decisiones en el interior de las organizaciones debería tener orientación deliberada hacia la construcción de un futuro sostenible para las personas que la componen. Es decir, que a través de un conjunto de prácticas tradicionales de gerenciamiento de su talento humano, se deberían contemplar también variables de tipo ambiental y social a fin de obtener (incluso) un mejor rendimiento de su trabajo. Allí es donde indiscutiblemente tiene cabida la inclusión laboral como un insumo de la política de gestión de las organizaciones, y en donde también se perciben brechas en la investigación existente, toda vez que la medición del impacto de la sostenibilidad aún es incipiente en términos del verdadero efecto a largo plazo que se obtiene gracias a la aplicación de dichas practicas.

\section{REFERENCIAS}

Asselin, L. \& Anh, V. (2008). Multidimensional poverty and multiple correspondence analysis. Ottawa: International Development Research Centre.

Atkinson, A. B. (1998). Preface. En, A. B. Atkinson \& J. Hills. Exclusion, Employment and Opportunity. London: Centre for Analysis of Social Exclusion, London School of Economics.

Barnard, C. (1938). The functions of the executive. Cambridge, MA: Harvard University Press.

Blaisdell, B. (2012, November 14). new ways to engage employees, suppliers and competitors in CSR. Interviewed by Kruschwitz, N. Retrieved from http://sloanreview.mit.edu 
Bogliacino, F. (2014). A critical review of the technology-inequality debate. Suma De Negocios, 5(12), 124-135.

Botello, H., Pedraza, A.C. \& Contreras, O.E. (2014). Análisis empresarial de la influencia de las TIC en el desempeño de las empresas de servicios en Colombia. Barcelona, España: Consejo Latinoamericano de Administración -CLADEA-.

Bourdeau, J. \& Ramstad, P. (2005). Talentship, talent segmentation, and sustainability: a new HR decision science paradigm for a new strategy definition. Human Resource Management, 44(2), 129-136. doi:10.1002/hrm.20054

Brokaw, L. (July 13, 2012). Marks and Spencer's emerging business case for sustainability. Retrieved from http://sloanreview.mit.edu

Comisión de la Comunidad Europea (2001). Libro Verde. Fomentar un marco europeo para la responsabilidad social de las empresas. Bruselas: Comunidad Europea.

Contreras, O.E. \& Rozo, I. (2015). Teletrabajo y sostenibilidad empresarial. Una reflexión desde la gerencia del talento humano en Colombia. (In press). Suma de Negocios, $6(13)$.

Contreras, O.E., Vecino, C.E. \& Pedraza, A.C. (2013) Sustainable human resources management: a first approach. Rio de Janeiro, Brasil: Consejo Latinoamericano de Administración CLADEA.

De Haan, A. (1999). Exclusión social en la política y la investigación: operacionalización del concepto. En C. Corredor. Pobreza y Desigualdad. Reflexiones conceptuales y de medición. Bogotá: Antropos, CINEP.

Dempsey, N., Bramley, G., Power, S. \& Brown, C. (2009). The social dimension of sustainable development: defining urban social sustainability. Sustainable Development, 289-300. doi: $10.1002 /$ sd.417

Dugarova, E. \& Lavers, T. (2014). Social Inclusion and the Post-2015 Sustainable Development Agenda. S.1.: UNRISD.

Eames, M. \& Adebowale, M. (2002). Sustainable development and social inclusion: towards an integrated approach to research (1st. ed.). York: Joseph Rowntree Foundation.

Ehnert, I., Harry, W. \& Zink, K. (2014). Social sustainability and quality of working life. In Sustainability and human resource management: Developing sustainable business organizations (1st ed.), (p. 126). Berlin, Heidelberg: Springer Verlag. 
La inclusión social de la fuerza laboral en Colombia. ¿En contravía de lo sostenible?

Orlando E. Contreras - Aura C. Pedraza Avella - Carolina Herrera Gómez

European Council. (2002). Revised text on the common objectives: fight against poverty and social exclusion: common objectives for the Second Round of National Action Plans. Bruselas: European Council.

Farné, S. (2003). Estudio sobre la calidad del empleo en Colombia. Lima: OIT.

Fiedler, F. \& Chemers, M. (1985). Liderazgo y administración efectiva. México: Trillas.

Freeman, C. (1990). The economics of innovation (Vol. 2). UK: Edward Elgar Pub.

Friedman, M. (1970, September 13). The social responsibility of business is to increase its profits. New York Times Magazine.

Friedman, A. (1970). Foundations of modern analysis. S.1.: Courier Corporation.

Fritz, M. \& Koch, M. (2014). Potentials for prosperity without growth: ecological sustainability, social inclusion and the quality of life in 38 countries. Ecological Economics, 191-199. doi:10.1016/j.ecolecon.

Gundogan, N., Bicerli, K. \& Aydin, U. (2005). The working poor: a comparative analysis. Eski ${ }^{\circ}$ ehir: Anadolu University.

Hart, S. (2005). Capitalism at the crossroads: the unlimited business opportunities in solving the world's most difficult problems. Upper Saddle River, NJ: Pearson Education.

Ickis, J.C., Prado, A. \& García-Rada, X. (2012). Florida Ice and Farm: sustainability champion from an emerging economy (A). Case 0179 INCAE Business School. Nicaragua: IESE Publishing LATAM.

ISO International Organization for Standardization (2010). Norma técnica de responsabilidad social ISO 26000. (1 ed.). Ginebra: ISO.

Jones, D.A. (2010). Does serving the community also serve the company? Using organizational identification and social exchange theories to understand employee responses to a volunteerism programme. Journal of Occupational and Organizational Psychology, $83,857-878$.

Jones, D., Willness, C.R. \& Madey, S. (2012). Why are job seekers attracted by corporate social performance? Experimental and Field Tests of Three Signal-Based Mechanisms. Academy of Management Journal. In revisión: AMJ-2011-0848.R2.

Lafuente, A., Viñuales, V., Pueyo, R. \& Llaría, J. (2003). Responsabilidad social corporativa y políticas públicas. Madrid: Fundación Alternativas. 
Levitt, T. (1958, September-October). The dangers of social responsibility. Harvard Business Review, 41-50.

Lis, B. (2012). The relevance of corporate social responsibility for a sustainable human resource management: an analysis of organizational attractiveness as a determinant in employees' selection of a (potential) employer. Management Revue, 23(3- Special Issue: Recent Developments and Future Prospects on Sustainable Human Resource Management), 279-295.

Marshall, A. (1938). Principles of Economics. (8 ed.). London: Macmillan.

McWilliams, A., Siegel, D.S. \& Wright, P. (2006). Corporate social responsibility: strategic implications. Journal of Management Studies, Wiley Online Library. DOI: $10.1111 / \mathrm{j} .1467-6486.2006 .00580 . x$

Millenium 3 (2007). Exclusión social y mercado laboral. Zaragoza, Aragón: Instituto Aragonés de Empleo.

Organización Mundial del Trabajo (2006). Iniciativa InFocus sobre responsabilidad social de la empresa. Ginebra: OIT.

Page, A. \& Katz, R.A. (2012, Fall). The Truth about Ben \& Jerry's. Stanford Social Innovation Review.

Pedraza, A. (2010). Exclusión social y segmentación laboral en Colombia. Tesis doctoral Ciencias Económicas. Universidad Nacional de Colombia, Facultad de Economía, Bogotá, Colombia.

Pedraza, A. (2011). Segmentación laboral en Colombia durante el período 2001-2006. Revista CIFE, 18(13), 101-123.

Pedraza, A. (2012). Exclusión social y empleo: ¿qué ocurre cuando hay segmentación laboral? Sociedad y Economía, (22), 135-162.

Pinillos, A.A. \& Fernández, J.L. (2011). De la RSC a la sustentabilidad corporativa: una evolución necesaria para la creación de valor. Harvard DEUSTO Business Review.

Porter, M.E. \& Kramer, M.R. (2006). Estrategia y sociedad: el vínculo entre ventaja competitiva y responsabilidad social corporativa» Harvard Business Review AL.

Porter, M.E. \& Kramer, M.R. (2011). La creación de valor compartido: cómo reinventar el capitalismo y liberar una oleada de innovación y crecimiento. Harvard Business Review AL, 33-49. 
La inclusión social de la fuerza laboral en Colombia. ¿En contravía de lo sostenible?

Orlando E. Contreras - Aura C. Pedraza Avella - Carolina Herrera Gómez

Poschen, P. (2012). Working towards sustainable development. Opportunities for decent work and social inclusion in a green economy. (1st. ed.). Geneva: International Labour Office.

Renwick, D.W.S., Redman, T. \& Maguire, S. (2013). Green human resource management: a review and research agenda. International Journal of Management Review. DOI: 10.1111/j.1468-2370.2011.00328.x

Rubio, M. J. \& Monteros, S. (2002). La exclusión social. Teoría y práctica de la intervención. Madrid: CCS.

Sen A. (1985). A sociological approach to the measurement of poverty: a reply to Professor Peter Townsend. Oxford Economics Papers, (37), 669-676.

Sen A. (1993). Capability and well-being. En M. Nusbaum \& A. Sen. The Quality of life. Oxford: Oxford University Press.

Sen A. (2000). Social exclusion: concept, application and scrutinity. Office of Environment and Social Development. Social Development Papers No. 1. Manila: Asian Development Bank.

Silver, H. (1995) Reconceptualizing social disadvantage: three paradigms of social exclusion. In G. Rodgers, C. Gore \& J.B. Figueiredo (eds.) Social exclusion: rhetoric, reality, responses (pp.58-80). Geneva: Institute of International Labour Studies.

Spreizer, G. \& Porath, C. (2012). Creating sustainable performance. Harvard Business Review.

Stone, D. \& Deadrick, D. (2015). Challenges and opportunities affecting the future of human resource management. Human Resource Management Review, 25, 139-145. doi:10.1016/j.hrmr.2015.01.003

Tarique, I. \& Schuler, R. (2010). Global talent management: Literature review, integrative framework, and suggestions for further research. Journal of World Business, 45(2), 122-133. doi:10.1016/j.jwb.2009.09.019

Ulrich, D. (1997). Human resource champions: the next agenda for adding value and delivering results. (1st. Ed.). USA: Harvard Business School Press.

Wernerfelt, B. (1984). A resource based view of the firm. Strategic Management Journal, 5 , 171-80.

WHO The World Health Organization (1995). Quality of life assessment: position paper from the world. Soc Sci Med, 41, 1403-1409. 